University of Wollongong

Research Online

Australian Institute for Innovative Materials -

Papers

Australian Institute for Innovative Materials

$1-1-2014$

Significant tunability of thin film functionalities enabled by manipulating magnetic and structural nano-domains

Igor A. Golovchanskiy

University of Wollongong, ig684@uowmail.edu.au

Alexey V. Pan

University of Wollongong, pan@uow.edu.au

Sergey A. Fedoseev

University of Wollongong, sf477@uowmail.edu.au

Michael Higgins

University of Wollongong, mhiggins@uow.edu.au

Follow this and additional works at: https://ro.uow.edu.au/aiimpapers

Part of the Engineering Commons, and the Physical Sciences and Mathematics Commons

Research Online is the open access institutional repository for the University of Wollongong. For further information contact the UOW Library: research-pubs@uow.edu.au 


\title{
Significant tunability of thin film functionalities enabled by manipulating magnetic and structural nano-domains
}

\author{
Abstract \\ The influence of laser frequency on the structure and physical properties of thin films grown by pulsed \\ laser deposition has been studied. Different types of thin films, hard ferromagnetic FePt L10 and quasi- \\ single crystal superconducting YBa2Cu3O7 (YBCO), have been used for demonstration of the effect. \\ Significant structural modifications have been obtained for the films with similar thicknesses. These \\ modifications are shown to dramatically control their corresponding properties, providing an instrumental \\ ability for tuning the practical characteristics of the films by changing the laser frequency of their \\ deposition. In particular, 20-fold increase of coercive field and modification of demagnetization \\ mechanism are obtained for FePt films by varying the frequency from $1 \mathrm{~Hz}$ to $6 \mathrm{~Hz}$. Over a similar \\ frequency range, a strong dependence on the laser frequency is discovered for the YBCO films for the \\ critical current density behavior as a function of the applied magnetic field [ $\mathrm{Jc}(\mathrm{Ba})]$ with the unexpected \\ reversal of $\mathrm{Jc}(\mathrm{Ba})$ curves with temperature. The mechanisms of structure modifications and \\ corresponding property variations are proposed.

\section{Keywords} \\ enabled, functionalities, magnetic, film, structural, thin, tunability, significant, nano, domains, manipulating \\ Disciplines \\ Engineering | Physical Sciences and Mathematics

\section{Publication Details} \\ Golovchanskiy, I. A., Pan, A. V., Fedoseev, S. A. \& Higgins, M. (2014). Significant tunability of thin film \\ functionalities enabled by manipulating magnetic and structural nano-domains. Applied Surface Science, \\ 311 549-557.
}




\title{
Significant tunability of thin film functionalities enabled by manipulating magnetic and structural nano-domains
}

\author{
Igor A. Golovchanskiy, Alexey V. Pan*, and Sergey A. Fedoseev \\ Institute for Superconducting and Electronic Materials, \\ University of Wollongong, Northfields Avenue, \\ Wollongong, NSW 2522, Australia \\ *Email: pan@uow.edu.au \\ Michael Higgins \\ Intelligent Polymer Research Institute, University of Wollongong, \\ Northfields Avenue, Wollongong, NSW 2522, Australia
}

\begin{abstract}
The influence of laser frequency on the structure and physical properties of thin films grown by pulsed laser deposition has been studied. Different types of thin films, hard ferromagnetic FePt $L 1_{0}$ and quasi-single crystal superconducting $\mathrm{YBa}_{2} \mathrm{Cu}_{3} \mathrm{O}_{7}$ (YBCO), have been used for demonstration of the effect. Significant structural modifications have been obtained for the films with similar thicknesses. These modifications are shown to dramatically control their corresponding properties, providing an instrumental ability for tuning the practical characteristics of the films by changing the laser frequency of their deposition. In particular, 20-fold increase of coercive field and modification of demagnetization mechanism are obtained for FePt films by varying the frequency from $1 \mathrm{~Hz}$ to $6 \mathrm{~Hz}$. Over a similar frequency range, a strong dependence on the laser frequency is discovered for the YBCO films for the critical current density behaviour as a function of the applied magnetic field $\left[J_{c}\left(B_{a}\right)\right]$ with the unexpected reversal of $J_{c}\left(B_{a}\right)$ curves with temperature. The mechanisms of structure modifications and corresponding property variations are proposed.
\end{abstract}

\section{INTRODUCTION}

Simple and reliable methods with the high degree of technological control are required for accurate adjustments of material structures and corresponding properties for the required performance. For physical vapour deposition (PVD) technologies, this control is conventionally achieved by manipulating interdependent parameters, such as temperature, atmosphere, flux of material, resultant film thickness ${ }^{1-6}$ and laser frequency ${ }^{6-10}$. In particular, the laser frequency for pulsed-based deposition (PLD) influences the film structure in many different ways, whose mechanisms can be contentious and not easily understood ${ }^{7-11}$. Thus, this interdependency is not trivial, impeding reproducibility and requiring sophisticated control with the help of, for example, reflection high-energy electron diffraction (RHEED) technique.

This work is focused on tuning the structure and properties of thin films through the variation of laser frequency. It turns out that the laser frequency is convenient and powerful tool for applications in thin film technologies.

Two model materials are chosen to study the influence of the laser frequency: $L 1_{0} \mathrm{FePt}$ hard ferromagnetic thin films and quasi-single-crystal superconducting $\mathrm{YBa}_{2} \mathrm{Cu}_{3} \mathrm{O}_{7}$ (YBCO) thin films. It should be noted that these model films are completely different: (i) YBCO is a perovskite type metal-oxide, while $\mathrm{FePt}$ is a metal with fct superlattice; (ii) for the best performance, a FePt film is required to consist of well separated islands of tens to hundreds of nanometers large, whereas YBCO should have a quasi-single-crystal structure completely covering substrate and be as smooth as possible; (iii) FePt films grow via typical Volmer-Weber island growth ${ }^{9,12}$, while YBCO is grown by Stranski-Krastanov mechanism ${ }^{13-15}$ as the result of the film composition and the crystal lattice mismatch with the substrate $\left(\mathrm{SrTiO}_{3}\right.$, in our case).

Quasi-single-crystal $c$-axis oriented epitaxial YBCO thin films are known to have the highest critical current density $\left(J_{c}\right)$ among high-temperature superconductors at temperature $T=77 \mathrm{~K}^{16,17}$. It makes $\mathrm{YBCO}$ thin films a perfect candidate for various electric power applications ${ }^{18-20}$. In addition, applications of YBCO thin films are associated with microwave electronic devices $^{21,22}$ and low signal electronic devices ${ }^{19,23,24}$. The critical current density of these films (like any type-II superconducting material) is governed by pinning of superconducting vortices by crystal defects. The strongest pinning sites consist of columns of a non-superconducting material with the diameter close to coherence length, and that are aligned with the direction of applied magnetic field $\left(B_{a}\right)^{25-27}$. For high quality YBCO thin films made by physical vapour deposition techniques, the out-of-plane edge dislocations are alike columns, which are formed in situ during growth of the films ${ }^{27}$. The arrays of these dislocations form low-angle domain boundaries ${ }^{13,15,28-30}$. A very large density of these pinning sites is required for high critical current densities. On the other hand, these pinning sites, created by disturbed crystal lattice parameters, possess suppressed superconducting order parameter. The superconducting order parameter defines the capability of the material to form the superconducting charge carriers (Cooper pairs). Hence, an optimal oxygen content and the best possible crystallographic order of YBCO films are necessary to reach high and homogeneous superconducting order pa- 
rameter (see, for example ${ }^{31,32}$ ). Various conditions relevant to applications, such as external magnetic fields and temperatures, can strongly affect the order parameter and pinning properties, which in turn can strongly influence the superconducting performance. Thus, fine tuning of deposition conditions is required to reach the best performance in particular conditions (for particular applications), where the deposition frequency can play the key role.

Furthermore, a critical thickness for the optimal YBCO performance exists ${ }^{5,6,33,34}$. In thicker films, the surface becomes rough and their properties degrade, unless a multilayered structure is introduced ${ }^{35}$. In thinner films, the integral capabilities of the films are reduced. The optimal thickness, defined at the maximum of the critical current density $\left(J_{c}\right)$ as the function of the film thickness ${ }^{5,33}$, is usually obtained at the deposition stage with all YBCO islands coalesced ${ }^{5,6,34}$. In this work, it is shown that the island density and the island growth kinetics can be effectively varied by changing deposition conditions (in particular, laser frequency), thus the optimal thickness can be adjusted at will.

FePt thin films with $L 1_{0^{-}} f c t$ superstructure are considered for various existing and emerging applications, such as perpendicular magnetic recording media (MRM) by Seagate $^{36}$, microelectromechanical systems (MEMS) ${ }^{37}$, ultra strong permanent magnets ${ }^{1,2,38}$, or ferromagneticsuperconducting heterostructures ${ }^{39}$. These applications become possible due to the film ability to form thermally stable islands (or grains) down to a few nanometers ${ }^{38,40,41}$ (boosting recording density ${ }^{42}$ ), their high magnetic anisotropy, very high coercivity (exceeding $10 \mathrm{~T})^{1}$ and magnetic energy product, the out-of-plane magnetization, and high corrosion resistance. The strong magnetic properties of the films can also be employed for the compensation of applied magnetic field induced effects $^{39}$ in certain architectures combining ferromagnets and superconductors ${ }^{43,44}$. The behaviour resulting from the interactions can extend magnetic screening (Meissner effect $)^{45}$ and even enhance current carrying ability in superconducting wires ${ }^{46}$.

In this work, a unique advantage of pulsed laser deposition (PLD) technique in tuning desirable structures and properties of the FePt and YBCO epitaxial films is demonstrated. To the best of our knowledge, such dramatic structure variations and such a broad range of practically achievable physical properties, being tuned by means of mere laser frequency variation, have not been reported before. Note, the key instrumental feature for varying properties of the films is the ability to manipulate the in-plane structure of the films, while their thickness is kept constant. In the case of YBCO thin films, it allows one to adjust critical current density dependence on applied magnetic field $\left(J_{c}\left(B_{a}\right)\right)$ for different applications at different operating temperatures. In the case of FePt thin films, it facilitates the adjustment of the coercive field and magnetic domain structure. Our new approach is in contrast to the commonly used method for the modulation of island structure of the FePt films by means of the thickness variation, which is proportional to the size of the islands ${ }^{1-3}$. However, making these films too thin may reduce the read-back signal below the detectable threshold and the magnetic energy product for permanent magnets.

\section{EXPERIMENTAL DETAILS}

\section{A. PLD of YBCO and FePt thin films}

Quasi-single-crystal c-oriented YBCO thin films were produced on $5 \times 5 \mathrm{~mm}$ size $\mathrm{SrTiO}_{3}$ (STO) substrates using standard pulsed-laser deposition (PLD) ${ }^{33,35}$. Deposition was performed by $\mathrm{KrF}$ excimer laser with the wave-length of $248 \mathrm{~nm}$. The laser fluency was kept at $\sim 3-4 \mathrm{~J} / \mathrm{cm}^{2}$, but taking into account optical path losses and laser beam homogenization, the fluency of $\sim 2 \mathrm{~J} / \mathrm{cm}^{2}$ is a more realistic estimation. The frequency of the laser was varied from 1 to $8 \mathrm{~Hz}$.

For the deposition of YBCO films, the PLD chamber was pre-evacuated down to $10^{-6}$ Torr. During the deposition, the substrate temperature and background oxygen pressure were $780^{\circ} \mathrm{C}$ and 300 mTorr, respectively. Distance between the substrate and the target was $85 \mathrm{~mm}$. The films where annealed in oxygen atmosphere at $400^{\circ} \mathrm{C}$ for one hour after deposition for oxygenation. The thin films measured in this work are of optimal thickness $d_{p} \simeq 300 \mathrm{~nm}$ thick $^{5,6,33}$, measured by Dektak profiler. The critical temperature was $T_{c} \simeq 90.0 \pm 0.5 \mathrm{~K}$ for films deposited with $5 \mathrm{~Hz}$ and $8 \mathrm{~Hz}$ frequencies and $T_{c} \simeq 91.5 \pm 0.5 \mathrm{~K}$ for $1 \mathrm{~Hz}$ deposited film, measured by DC magnetic measurements in PPMS at $B_{a}=2.5 \mathrm{mT}$.

FePt thin films with $L 1_{0} f c t$-oriented islands were deposited on the same type of the STO substrates using the same PLD system. The justification for the substrate material choice can be found elsewhere (see Ref. ${ }^{11}$ and references therein). The base pressure in the PLD chamber was typically held at $\leq 10^{-8}$ Torr that was reached after additional PLD chamber cleaning and baking routine. The pulsed laser deposition of the films was carried out with the substrate temperature held at $800^{\circ} \mathrm{C}$, which degraded vacuum during deposition down to $\sim 5 \times 10^{-7}$ Torr due to thermal desorption from the heater. The deposition temperature was chosen to ensure that the $L 1_{0}$ structure of the FePt alloy forming at $500^{\circ} \mathrm{C}^{47-50}$ was complete ${ }^{2,37,50-53}$. The deposition process, facilitating almost equiatomic composition in the FePt epitaxial superstructures ${ }^{54,55}$, has been described elsewhere $^{11}$. The deposition frequency was varied from $1 \mathrm{~Hz}$ to $6 \mathrm{~Hz}$, so that the resultant thickness of the films was always approximately $20 \mathrm{~nm}$. 


\section{B. Measurement and microscopy techniques}

Magnetization measurements were carried out on Quantum Design Vibrating Sample Magnetometer Physical Properties Measurement System (VSM PPMS) with the magnetic field applied perpendicular to the surface of the films. For magnetization dependence on applied magnetic field measurements magnetic field sweep rate was kept at $5.0 \mathrm{mT} / \mathrm{s}$. The frequency of VSM was $40 \mathrm{~Hz}$ and the amplitude of vibrations was $2 \mathrm{~mm}$ for YBCO samples and $1 \mathrm{~mm}$ for FePt samples. The dependence of YBCO measured superconducting properties on VSM settings and magnetic field sweep rate can be found in Refs. ${ }^{56,57}$. The $J_{c}$ of YBCO films was determined from the magnetization measurements using Bean formula for rectangular samples $^{58}: J_{c}=2 \Delta M /\left[w_{p}\left(1-w_{p} / 3 l_{p}\right)\right]$ in $\mathrm{A} / \mathrm{m}^{2}$, where $w_{p}$ and $l_{p}$ are respectively width and length of the samples measured, $\Delta M$ is the opening of hysteresis loops per unit volume. For YBCO films, $l_{p}=w_{p}=5 \mathrm{~mm}$.

Atomic Force Microscopy (AFM) imaging of $\mathrm{YBCO}$ films was performed using an MFP-3D Asylum AFM (Asylum Research) in $\mathrm{AC}$ mode using $\mathrm{Al}$ reflex coated silicon probes with approximately $330 \mathrm{kHz}$ resonance frequency and $42 \mathrm{~N} / \mathrm{m}$ spring constant (NCHR probes, Asylum Research). AFM and Magnetic Force Microscopy (MFM) of FePt films was performed using the same instrument in MFM mode using $\mathrm{CoCr}$ coated silicon probes with approximately $70 \mathrm{kHz}$ resonance frequency, $2 \mathrm{~N} / \mathrm{m}$ spring constant and coercivity of $0.04 \mathrm{~T}$ (ASYMFM probes, Asylum Research). The probes were magnetized by placing near a permanent magnet for $15-30$ secs. During MFM, an AC mode scan was first performed along a single line to record topography after which the tip was raised above the surface (termed Nap mode, approximately $15 \mathrm{~nm}$ height) and scanned along the same line, while measuring the phase signal of the cantilever in response to the magnetic force interactions between the tip and sample. Thus, corresponding topography and MFM (phase) images were collected simultaneously from the scan area. All images were taken in air.

\section{TUNING SURFACE ARCHITECTURE AND PROPERTIES}

\section{A. FePt thin films}

Epitaxial FePt thin films deposited at different laser frequencies (Fig. 1) indicate Volmer-Weber 3D island growth mechanism typical for metals grown on insulators ${ }^{9,11,12}$. The films deposited at the laser frequency of $6 \mathrm{~Hz}$ exhibit a fine well-defined rectangular island architecture with rather narrow size and shape distributions, and marginal island coalescence [Figure 1(c)]. In Fig. 2, the island size distribution for the $2 \mathrm{~Hz}$ and $6 \mathrm{~Hz}$ films are shown. As can be seen, the average island size of the $6 \mathrm{~Hz}$ films is $\sim 45 \mathrm{~nm}$. The $2 \mathrm{~Hz}$ film [Figure 1(b)] demonstrates the structure with larger elon- gated coalescent islands, but no island percolation occurred. The average size of elongated islands of the $2 \mathrm{~Hz}$ film is $\sim 100 \mathrm{~nm}$ (Fig. 2). The $1 \mathrm{~Hz}$ film [Figure 1(a)] exhibits a clear percolated surface structure resulted from heavy amalgamation of the elongated islands [shown in Figure 1(b)] during the growth process. Obviously, these stages of the film growth (small islands, coalescence, elongation, percolation and hole-filling) follow one after another as the thickness increases and more material is deposited on the substrate. Strikingly, the uniqueness of the structures shown in Fig. 1 is that all these stages are achieved by changing the deposition frequency only while the thickness remains the same. However, the thickness range at which all these growth stages can be obtained by varying the frequency is quite narrow.

The increase of the island density with increasing deposition frequency observed in Fig. 1 is well consistent with the key result obtained in Ref. ${ }^{11}$ that the laser frequency affects the ratio of the out-of-plane to the in-plane growth. The decrease of in-plane to out-of-plane growth rates ratio at high surface coverage with increasing frequency leads to delayed impingement and coalescence of the islands and, as a consequence, to a structure with smaller islands. Obviously, the thickness would also be sensitive to the ratio changes, which is shown to be a critical factor in the film growth transitions ${ }^{2,11}$. The advantage of this growth mechanism is that the in-plane and out-of-plane growths of the islands and/or grains upon PLD process can be manipulated nearly independently, allowing the desired tunability, which is the central result of this work.

The properties of FePt films appear to be tailored by the island structure. Indeed, the $1 \mathrm{~Hz}$ film, consisting of the large percolative islands [Figure 1(a)], demonstrates a narrow hysteresis loop with a small coercive field $H_{c} \simeq 0.1 \mathrm{~T}$ [Figure 3]. These magnetic properties are the consequence of the quasi-continuous surface structure, because it creates a possibility for the formation of magnetic domain walls and negligible pinning for their propagation (as confirmed by MFM studies below). The qualitatively different surface patterns of the films, obtained by deposition at higher frequencies [Figure 1(b,c)], exhibit a drastic difference in the magnetic behaviour with very large coercive fields about $\sim 4.7 \mathrm{~T}$ (Figure 3 ). These hysteresis loops are typical for highly ordered $f c t$ FePt island structures with the easy magnetization axis of the films being out-of-plane. The coercivity fields of the $2 \mathrm{~Hz}$ and $6 \mathrm{~Hz}$ films are identical despite of drastic differences in their structures [Figs. 1(b,c) and 2]. Moreover, the $H_{c}$ of these films is identical over the entire temperature range of the measurements [from $300 \mathrm{~K}$ to $4.2 \mathrm{~K}$ (inset in Figure 3)], indicating similar magnetic anisotropy $K_{u}$.

In case of equal $H_{c}$ of specimens with different structures (the case of $2 \mathrm{~Hz}$ and $6 \mathrm{~Hz}$ deposited films) the influence of the film structure on magnetic properties can be accentuated by performing a switching field distribution (SFD) measurement. In Ref. ${ }^{11}$, it was demonstrated that 

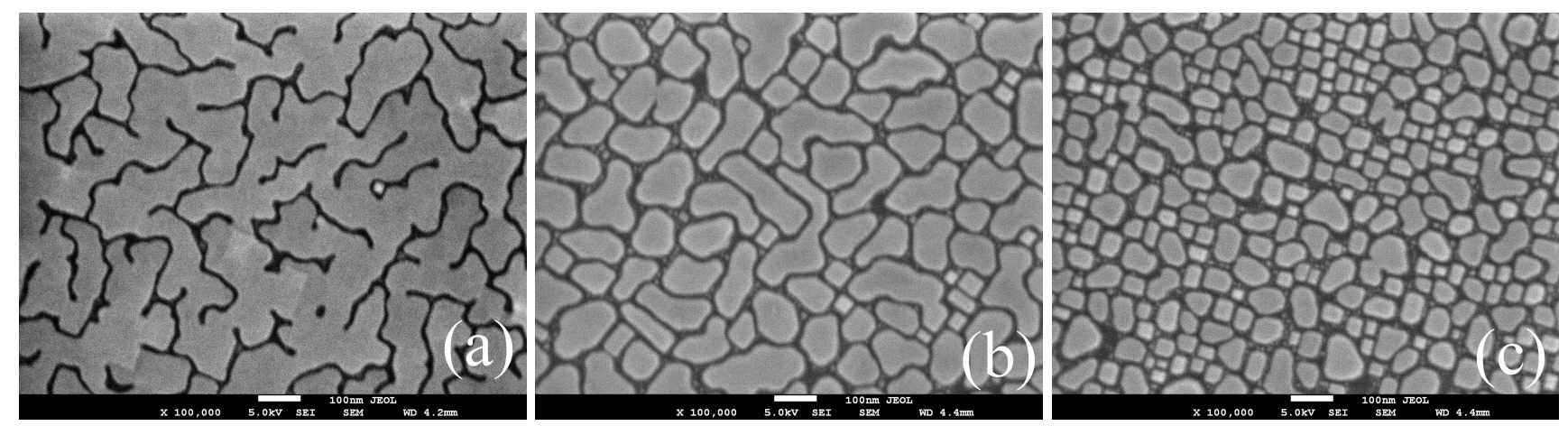

FIG. 1. Scanning electron microscopy images of $1 \mathrm{~Hz}$ (a), $2 \mathrm{~Hz}$ (b) and $6 \mathrm{~Hz}$ (c) deposited FePt films.

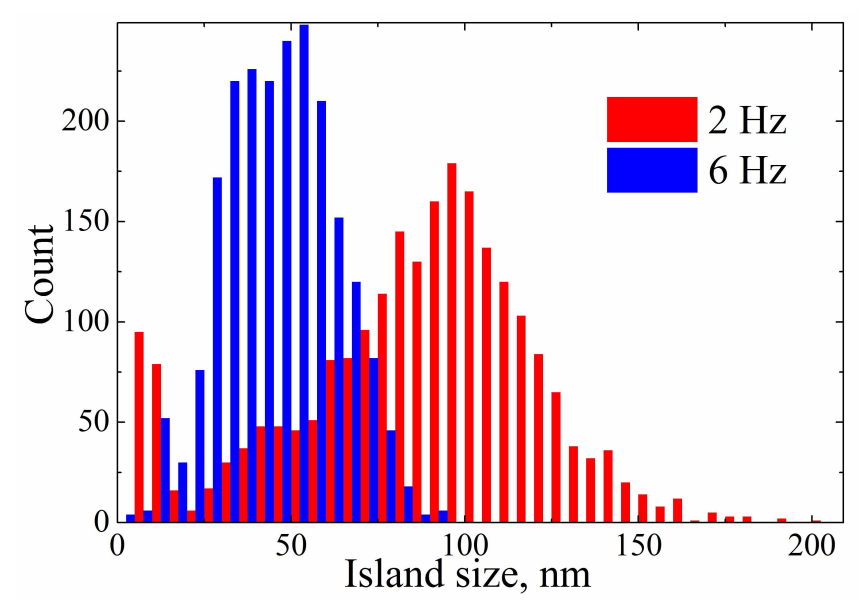

FIG. 2. Island size distribution for the films deposited at $2 \mathrm{~Hz}$ and $6 \mathrm{~Hz}$. The island size was defined as a square root of the measured island areas.

SFD of FePt films with identical $H_{c}$ reveal strong dipolar interactions between islands around the coercive field. However, the amplitude of the interaction appears to be proportional to the average island size. A lower level of the interactions between the islands indicates that the structure obtained at $6 \mathrm{~Hz}$ can be more suitable for some applications than the one obtained at $2 \mathrm{~Hz}$.

The magnetic domain structure for the films was studied by AFM and MFM, in order to correlate the magnetic structure with the surface morphology. Before imaging, the FePt samples were magnetized in PPMS at $B_{a}=-8 \mathrm{~T}$ to obtain uniform negative magnetization through the film, and then demagnetized by applying the field equal to the coercive field value (at $0.1 \mathrm{~T}$ for the $1 \mathrm{~Hz}$ film, at $4.6 \mathrm{~T}$ for the $2 \mathrm{~Hz}$ and $6 \mathrm{~Hz}$ films) to have the parity of positively and negatively magnetized areas. Fig. 4 shows MFM images (a-c) and overlayed images of MFM with their corresponding topographic AFM images (d-f) of the FePt thin films deposited at different frequencies. The dark and bright parts in the images represent the magnetic film domains with upward and downward

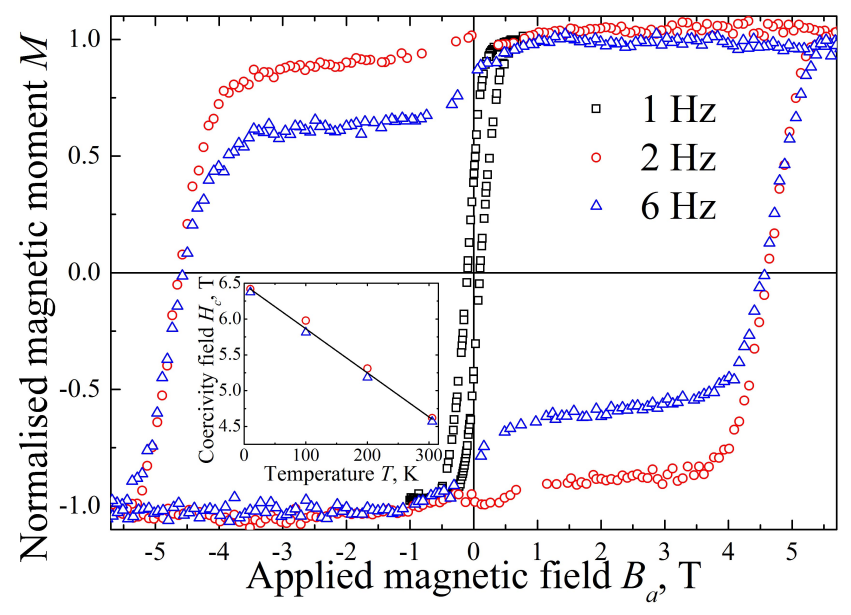

FIG. 3. Hysteresis loops of the FePt films deposited at different frequencies with the magnetic field applied perpendicular to the surface of the films. The inset shows the coercive field dependence on temperature for the $2 \mathrm{~Hz}$ and $6 \mathrm{~Hz}$ films.

magnetizations, respectively.

Single domain (SD) structure is obtained for both $2 \mathrm{~Hz}$ and $6 \mathrm{~Hz}$ films [Figs. 4(b) and (c), respectively], the SD structure implies that the shape of magnetic domains match exactly the shape of the FePt islands [Figs. 4(e) and (f)]. All SD regions are slightly lighter (or darker) towards their edges due to the flux compensation between the opposite magnetic fluxes from the domains with opposite magnetization at the island periphery [Fig. 4(b,c)]. The level of grey tone may differ for different domains due to different island volumes and/or composition variation resulting in their different magnetizations. The demagnetization in the samples with the SD structure is dominated by the spontaneous flip of the magnetization orientation, assisted by a high out-of-plane magnetic anisotropy. This demagnetization process is responsible for the high coercive fields. Magnetic landscape recorded for the $6 \mathrm{~Hz}$ film [Fig. 4(c)] indicates the capability for a further enhancement of the resolution for applications in magnetic recording media. 

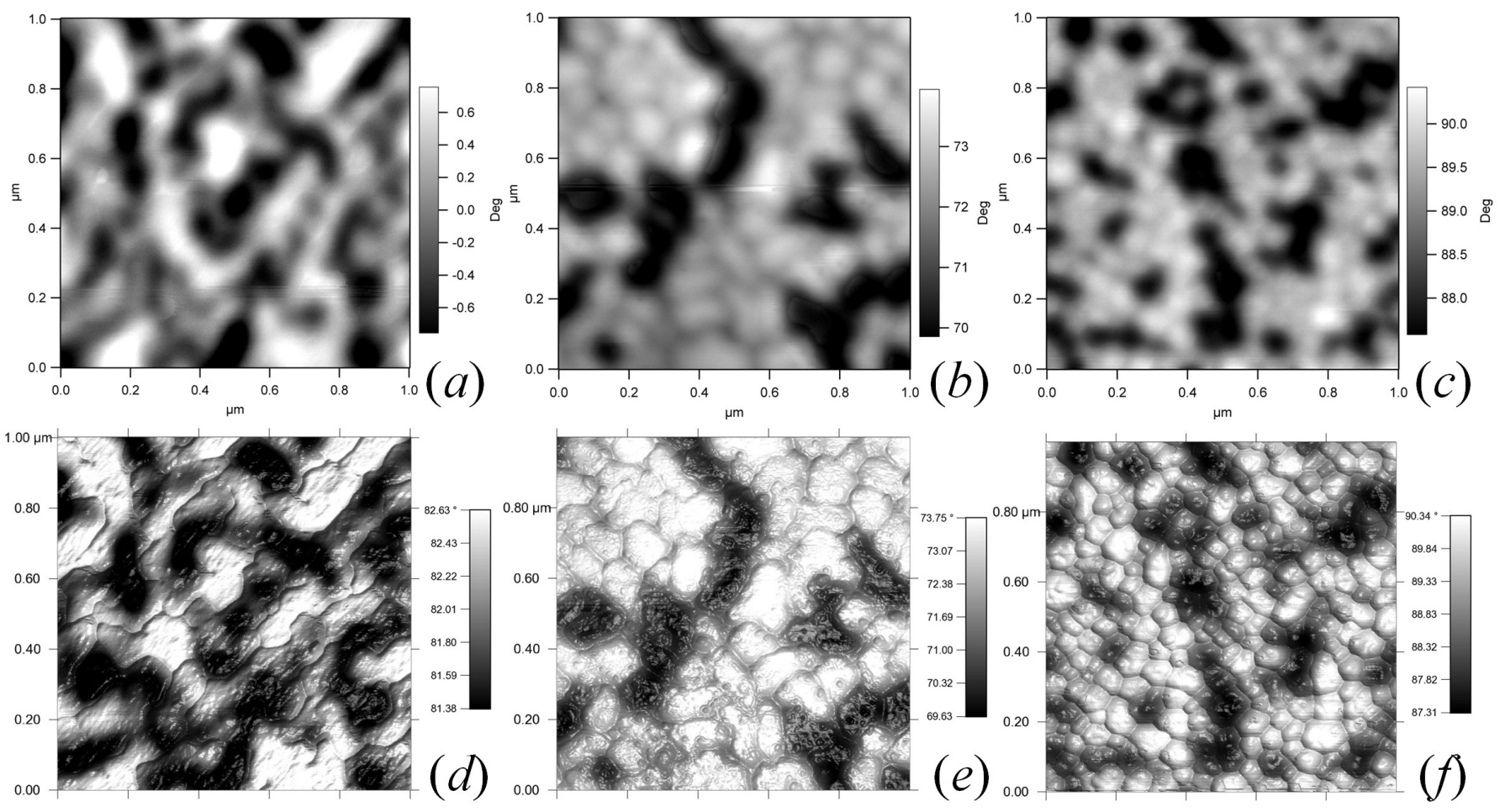

FIG. 4. Magnetic force microscopy (a,b,c) and corresponding AFM overlayed with MFM (d,e,f) images of the $1 \mathrm{~Hz}(\mathrm{a}, \mathrm{d}), 2 \mathrm{~Hz}$ (b,e) and $6 \mathrm{~Hz}(\mathrm{c}, \mathrm{f}) \mathrm{FePt}$ films.

The multiple magnetic domains (uninterrupted by structural edges) can be identified in the $1 \mathrm{~Hz}$ film possessing so-called percolated surface structure [Fig. 4(a,d)]. Such multiple domain (MD) structure can also be found in the films with the island structure, if the size of the islands exceeds the critical size of the SD islands $\left(\sim 200 \mathrm{~nm}\right.$ for the in-plane direction $\left.{ }^{59,60}\right)$. The demagnetization process of the MD films is dominated by the nucleation of magnetization reversal followed by domain wall displacement, which is energetically favourable without structural pinning for the domain walls. It would explain the low coercive fields in these films, in spite of their high magnetic anisotropy.

Note, such magnetic structure variation was previously observed only in films with significantly different thicknesses ${ }^{59,60}$, implying corresponding structural differences required for the different states of the magnetic domain formation. In our case, the thickness of the films is identical. The structural differences and, hence, different magnetic domain formation driving the corresponding magnetic properties of the films are obtained through laser frequency adjustment, which emphasises the tuning efficiency of the laser frequency upon PLD.

\section{B. YBCO thin films}

The study of YBCO thin films properties in this section was done in films with an "optimal thickness" 5,6,33,34, which is determined to be of $\sim 300 \mathrm{~nm}$ at the highest $J_{c}\left(B_{a}\right)$ measured. The YBCO PLD films of the optimal thickness with the highest $J_{c}\left(B_{a}\right)$ are usually associated with rather advanced island structure ${ }^{61-63}$ with pronounced topology and rather high surface roughness ${ }^{6,33}$ (unless special techniques are employed to reduce it ${ }^{33,35}$ ). The developed structure and its corresponding topography hinder the study of the island growth and structure. Thus, the island structure of YBCO films as a function of the PLD laser frequency has been studied on thinner films, which have less developed surface topography. As the deposition is a continuous process, the surface structure and its variations detected on thinner samples sets the basis for the further film growth, hence it qualitatively reflects the changes of the film's properties as well.

As noted in the previous section, the film surface structure variation with changing laser frequency is extremely sensitive to deposition parameters and the thickness. Basically, it means that if such variation does take place it still can be "hidden" by an inappropriate thickness. To study the structure variation of YBCO thin films with changing the frequency, first an appropriate thickness of $25 \mathrm{~nm}$ was chosen to reveal the influence of the laser frequency variations as follows. The series of films with 
thicknesses from $10 \mathrm{~nm}$ to $50 \mathrm{~nm}$ was produced at different frequencies without annealing, necessary for oxygenation, in an attempt to exclude thermodynamically driven surface modifications after PLD.

No significant difference in the surface structure of the films deposited at different frequencies was observed in the films with thicknesses below $10 \mathrm{~nm}$ (below the islanding threshold thickness ${ }^{13,64,65}$ ). The difference could not be detected due to one of the following reasons. (i) The variation of the laser frequency does lead to changes in the surface structure of the films. However, the structures were modified during the post-deposition cooling from $780^{\circ} \mathrm{C}$ down to $500^{\circ} \mathrm{C}$ (which takes a few minutes) after switching off the heater, so that any possible differences faded away. As the thickness is small these modifications might be rather rapid at high temperatures. (ii) The different deposition frequency does not affect the structure below $10 \mathrm{~nm}$ because the films exhibit a $2 \mathrm{D}$ layer-by-layer growth mode at this thickness.

In the case of larger thicknesses (above $30 \mathrm{~nm}$ ), the structure variation could be observed but to a rather small extent, so that the observation could not be confirmed (possibly due to the too large amount of material being already deposited). Thus, the thickness of $25 \mathrm{~nm}$ was chosen for studying the effect of the laser frequency influence on the surface structure. Although, the postdeposition cooling did take several minutes, it most likely had little effect on the surface structure due to only short time being at high temperatures $\left(>500^{\circ} \mathrm{C}\right)$ with the material being already adhered to the substrate rather than being just above the surface in the plasma state.

It should again be emphasised that the films of the "optimal thickness" reveal strong topology/roughness dependence on laser frequency ${ }^{6,34}$. However, it is not straightforward how to interlink this dependence with their superconducting properties. The "overdeveloped" 3D structure of the films with high surface roughness makes the study of the island structure almost impossible. On the other hand, the top layers of the film with a significant thickness may have the least effect on the properties ${ }^{66}$. Thus, it is natural to associate the growth of $25 \mathrm{~nm}$ thick films with their superconducting properties, keeping in mind that the surface may still be one of the factors affecting the behaviour of the films ${ }^{33,35}$.

Fig. 5 shows AFM images of the typical surface structures in the $25 \mathrm{~nm}$ thick YBCO thin films deposited at $1 \mathrm{~Hz}$ [Figs. 5(a,b)] and $8 \mathrm{~Hz}$ [Figs. 5(c,d)]. These structures indicate Stranski-Krastanov growth mode, as expected for YBCO films on STO substrates by $\mathrm{PLD}^{13,15,62-64,67-69}$. After reaching the threshold thickness ${ }^{13,64,65}$, the film growth can be characterised as initial strain-driven islanding and subsequent islands growth in the presence of a wetting layer of $\mathrm{YBCO}$ on a substrate ${ }^{70}$. Depending on the growth rate, the $3 \mathrm{D}$ island growth can be in $2 \mathrm{D}$ growth mode at higher rates or due to spiral growth around screw dislocation at lower rates $^{62,63,71}$. In case of the $1 \mathrm{~Hz}$ film, it was not possible to distinguish whether the islands grow due to the spiral growth or 2D growth [Fig. 5(b)], while at the higher frequencies the island growth happens in 2D mode as can be seen by the stacks and individual flat platelets [Fig. 5(d)].

The growth analysis of the FePt thin films cannot be applied for YBCO thin films due to their different growth modes. Indeed, the FePt films demonstrate pronounced and even sharp edges of the islands that are perpendicular to the substrate surface (typical for Volmer-Weber mechanism); whereas in YBCO thin films, a significant number of islands are two-dimensional [Fig. 5(d)]. The ones that are three-dimensional have a pyramid-like shape with pronounced stack of terraces, in particular well seen in Fig. 5(b). Moreover, the presence of the wetting layer for YBCO growth is not considered in the model developed for FePt films in Ref. ${ }^{11}$. Thus, the film growth stages well-explained for FePt film growth are more sophisticated and are too difficult for quantitative analysis in the case of YBCO.

The film structure dependence on laser frequency variation can be analysed with a simple expression ${ }^{71}$

$$
L \sim\left(D_{s} / f\right)^{1 / 2},
$$

where $L$ is a $2 \mathrm{D}$ island size, $D_{s}$ is the surface diffusion of adatoms/clusters on a substrate (in our case, it is constant because deposition temperature, laser energy and duration of laser pulse are kept constant), $f$ is a laser frequency. In this expression, $L$ is simply a distance that adatom is able to travel before it will be immobilised by the next portion of deposited adatoms. Surface structure variation with laser frequency [Fig. 5] agrees with expression 1: the higher the frequency, the smaller the islands. In Fig. 5(a,b) for the $1 \mathrm{~Hz}$ film, large 3D pyramid-like islands can be clearly distinguished with the base size of $\sim 250 \mathrm{~nm}$. A higher degree of 3D islanding (typically, if 3D islands consist of 4-5 terraces) indicates that adatoms have had enough time between laser pulses for diffusion to distances up to $\sim 250 \mathrm{~nm}$ and for giving an impact on strain driven 3D islanding. In Fig. 5(c,d) for the $8 \mathrm{~Hz}$ film, the surface mainly consists of randomly distributed 2D islands with typical size $\sim 100-150 \mathrm{~nm}$. The level of 3D islanding is much less pronounced than for the $1 \mathrm{~Hz}$ film in Fig. 5(a,b). It indicates (according to Eq. 1) that amount of time between laser pulses was not enough for thermodynamically driven 3D island growth; the typical distance of adatom diffusion was $\sim 100 \mathrm{~nm}$. The structure of the $5 \mathrm{~Hz}$ film is an intermediate case, with a mixture of both $2 \mathrm{D}$ and $3 \mathrm{D}$ pyramid-like islands and island size $\sim 150-200 \mathrm{~nm}$. Island size variation with the laser frequency obtained experimentally consists well with the estimations from Eq. 1: experimentally, the size of islands decreases by a factor of $\sim 2.5$ with increasing laser frequency from $1 \mathrm{~Hz}$ to $8 \mathrm{~Hz}$, while the estimation from Eq. 1 provides a factor of $\sim 2.8$; for the frequency increase from $1 \mathrm{~Hz}$ to $5 \mathrm{~Hz}$ the island size decreases by a factor of 1.7, while from Eq. 1 it should be $\sim 2.2$; for the increase from $5 \mathrm{~Hz}$ to $8 \mathrm{~Hz}$ the decrease is by a factor of 1.5, while Eq. 1 gives $\sim 1.3$.

An indication of island structure variation may be ob- 

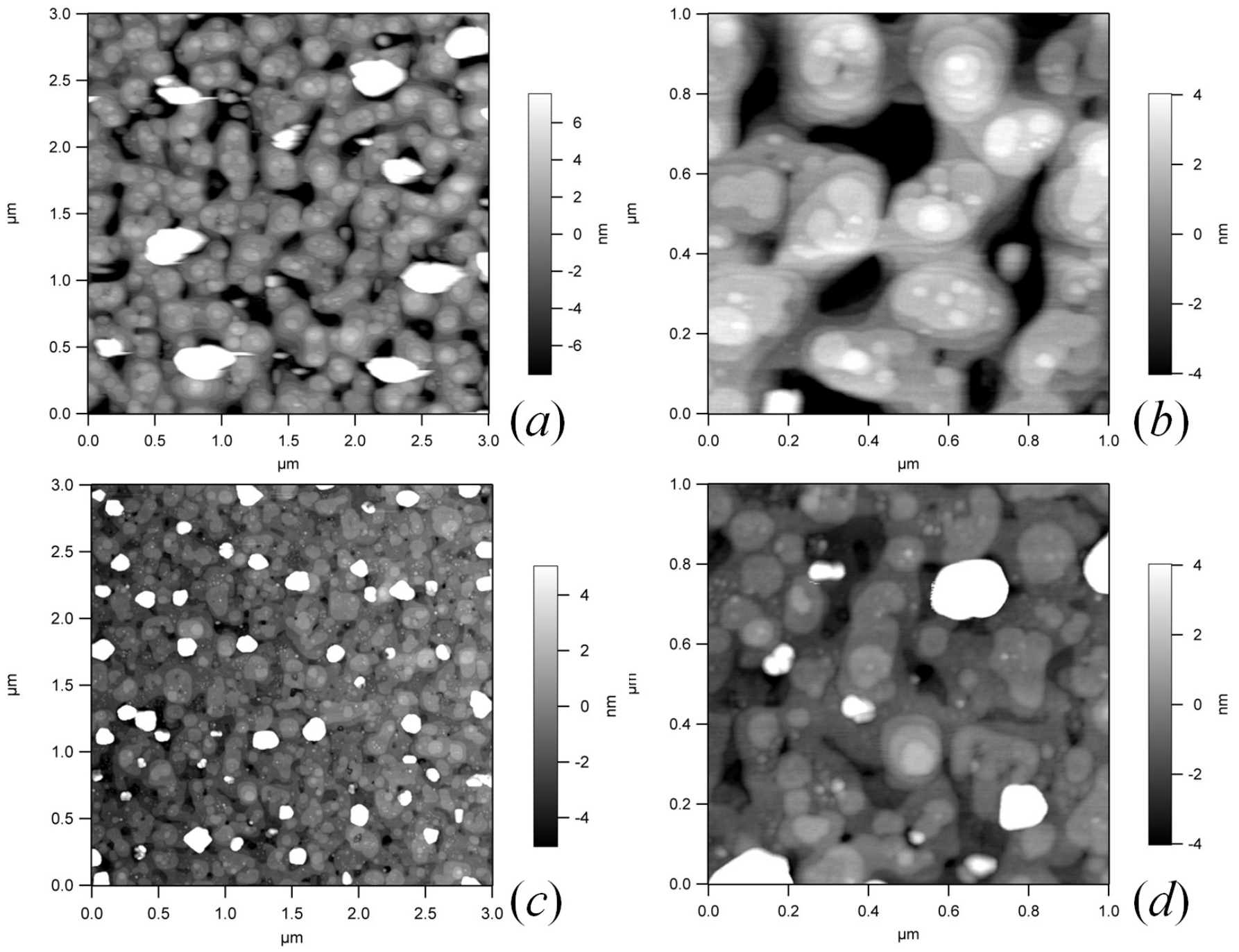

FIG. 5. The surfaces of the $25 \mathrm{~nm}$ thick YBCO films deposited at $1 \mathrm{~Hz}(\mathrm{a}, \mathrm{b})$, and $8 \mathrm{~Hz}(\mathrm{c}, \mathrm{d})$ obtained by atomic force microscopy.

tained from the comparison of the surface roughness obtained from the landscapes in topographical AFM images. In Table I, the results of roughness calculations on $3 \times 3 \mu \mathrm{m}^{2}$ scan area and the corresponding characteristic surface area ratio $(S)$ are presented. The roughness was calculated as root mean square (RMS) of the topography amplitude. $S$ was obtained from the ratio of the total surface area of the AFM topography to the scan area. These two surface parameters together can give an insight into YBCO island structure. From the point of view of the island structure, the systematic decrease of both RMS and $S$ with increasing deposition frequency (Table I) indicates the structure transition from larger and taller well separated islands to smaller and shorter closer located islands (the island density increases, the island height decreases).

Four basic qualitative conclusions can be made by analysing Fig. 5 and corresponding roughness parame-

\begin{tabular}{|c|c|c|}
\hline $\begin{array}{c}\text { Deposition } \\
\text { frequency } f, \mathrm{~Hz}\end{array}$ & $\begin{array}{c}\text { Roughness } \\
\text { RMS, nm }\end{array}$ & $S$ \\
\hline 1 & 3.122 & 1.0069 \\
\hline 5 & 2.238 & 1.0019 \\
\hline 8 & 1.713 & 1.0007 \\
\hline
\end{tabular}

TABLE I. Roughness parameters obtained from $3 \times 3 \mu \mathrm{m}$ AFM scan of YBCO thin films deposited at different frequencies (Fig. 5(a,c).

ters in Table I: (i) the density of the islands increases with laser frequency [best seen in Fig. 5(a,c)]; (ii) the roughness of the film surface (the degree of the $3 \mathrm{D}$ islanding) increases with decreasing laser frequency; (iii) as the higher degree of the $3 \mathrm{D}$ islanding is observed at lower deposition frequencies, it may indirectly indicate larger amount of accumulated strains due to lattice mis- 
fit at higher laser frequencies (it can lead to higher density of dislocation known to reduce strain); (iv) significantly lower degree of 3D islanding together with lower roughness and smaller $S$ of the films deposited at higher frequencies indicate an advanced in-plane island growth (unlike in the case of the FePt films for which higher frequencies promoted the out-of-plane growth).

We note two cases of potentially controversial results found in the literature, and propose plausible explanations lifting these contradictions.

First, in Refs. ${ }^{68}$ and $^{71}$, the laser frequency was varied by one order of magnitude (between 1 and $10 \mathrm{~Hz}$ and between 5 and $50 \mathrm{~Hz}$, respectively), but, in contrast to our results, it did not affect the growth mode or the island size (i.e., the structure of films did not obey Eq. 1). This controversy can be explained as follows. The surface diffusivity $D_{s}$ in Eq. 1 is predominantly determined by the plume dynamics (i.e., its kinetic energy), as well as by substrate temperature. The kinetic energy of adatoms on the substrate surface decreases roughly exponentially with increasing the distance from the target and the background pressure ${ }^{72,73}$. In Ref. ${ }^{68}$ and $^{71}$, the deposition was performed at the background oxygen pressure of $P_{\mathrm{O}_{2}} \simeq 112$ mTorr (which is by a factor of 2.7 lower than in our work), as well as with target-substrate distances of $55 \mathrm{~mm}$ in Ref. ${ }^{68}$ and $35 \mathrm{~mm}$ in Ref. ${ }^{71}$ (which are significantly smaller than in our work being $85 \mathrm{~mm}$ ). Therefore, the kinetic energy of adatoms in Refs. ${ }^{68}$ and $^{71}$ was substantially higher and resulted in larger diffusion coefficients $D_{s}$ than in our work. Thus, all diffusionguided processes (i.e., 3D island formation and growth) were rapid and likely completed between the laser pulses for the laser repetition rates studied in Refs. ${ }^{68}$ and $^{71}$. In contrast, the smaller $D_{s}$ enables us to effectively control the surface structure by varying time between laser pulses in agreement with Eq. 1.

Second, in Refs. ${ }^{4,34,68,74}$, films deposited at low repetition rates had smoother surfaces, which is in opposite to our results provided in Table I. However in those works, the roughness was studied on thicker films (> $200 \mathrm{~nm}$ ) with highly developed surface structures containing outgrowths, holes, pinholes and other defects formed due to incomplete island coalescence of relatively thick films ${ }^{4,34}$ or stress-guided surface roughening after complete coalescence ${ }^{5,33,35}$. Upon deposition on highly developed surface with low deposition rates adatoms have more time to diffuse to sites with high coordination number, reducing roughness as observed in Refs. ${ }^{4,34,68,74}$. In our work, the roughness (Table I) was measured in much thinner films, reflecting the dependence of StranskiKrastanov 2D/3D island structure on laser frequency.

The main superconducting properties of our YBCO films $\left(J_{c}\right.$ and $\left.T_{c}\right)$ can be easily tailored via their strong dependence on the structure and composition, which in turn can be controlled by varying the PLD frequency. Fig. 6 shows the zero-field cooled magnetization measurements at $B_{a}=2.5 \mathrm{mT}$ for the films deposited at $1 \mathrm{~Hz}, 5 \mathrm{~Hz}$ and $8 \mathrm{~Hz}$. The following values are ob-

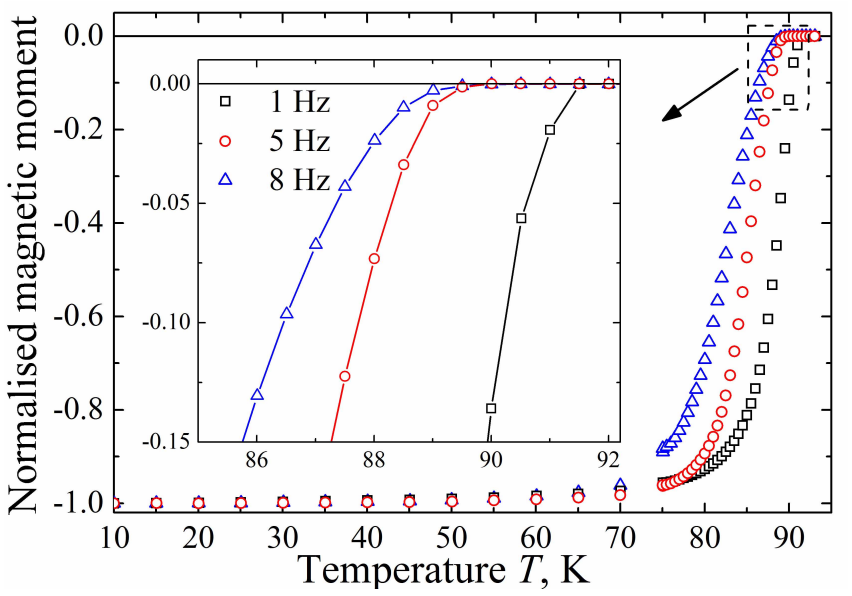

FIG. 6. Temperature dependence of magnetization measured at $B_{a}=2.5 \mathrm{mT}$ applied transverse to the surface of the films upon heating from the zero-field cooled state for the YBCO films deposited at different frequencies.

tained: $T_{c} \simeq 90.0 \pm 0.5 \mathrm{~K}$ for the $5 \mathrm{~Hz}$ and $8 \mathrm{~Hz}$ films and $T_{c} \simeq 91.5 \pm 0.5 \mathrm{~K}$ for the $1 \mathrm{~Hz}$ film, which are determined at the onset of the diamagnetic behaviour as shown in the inset to Fig. 6. The critical temperature decreases with increasing laser frequency. Higher $T_{c}$ of YBCO films deposited at lower frequency can be a result of longer oxygenation time between the laser pulses ${ }^{68}$. Furthermore, the $T_{c}$ transitions broadens with increasing laser frequency (Fig. 6). It can indicate a larger amount of non-superconducting phases or phases with weaker superconductivity, such as domain boundaries, dislocations, oxygen vacancies, non-stoichiometrical inclusions, lower lattice ordering, outgrowth, etc. Hence, it may be appropriate to conclude that the quality of YBCO thin film degrades with increasing the laser frequency of their deposition.

Critical current density is to a large extent determined by a number of vortex pinning sites (structural defects of the size of coherent length). These defects immobilize the flux-line lattice being driven by the Lorentz force if external current is applied. A moving flux-line lattice causes energy dissipation and, as a result, superconducting performance degradation. The best possible natural pinning centres in $\mathrm{YBCO}$ thin films are edge and screw dislocations, in particular for the fields applied parallel to these dislocations ${ }^{27,61,75}$. These dislocations are formed in the out-of-plane direction during pulsed laser deposition of the films ${ }^{28,61,65,75,76}$ as a result of the YBCO/STO crystal lattice misfit. In Fig. 7 and 8, the critical current density dependences on the applied magnetic field are shown for the films deposited at different laser frequencies.

In Fig. 7 , the $J_{c}\left(B_{a}\right)$ dependences are shown for three deposition frequencies at $10 \mathrm{~K}$. The critical current density increases at higher deposition frequencies, in particular well-seen above $1 \mathrm{~T}$. It indicates an increased num- 


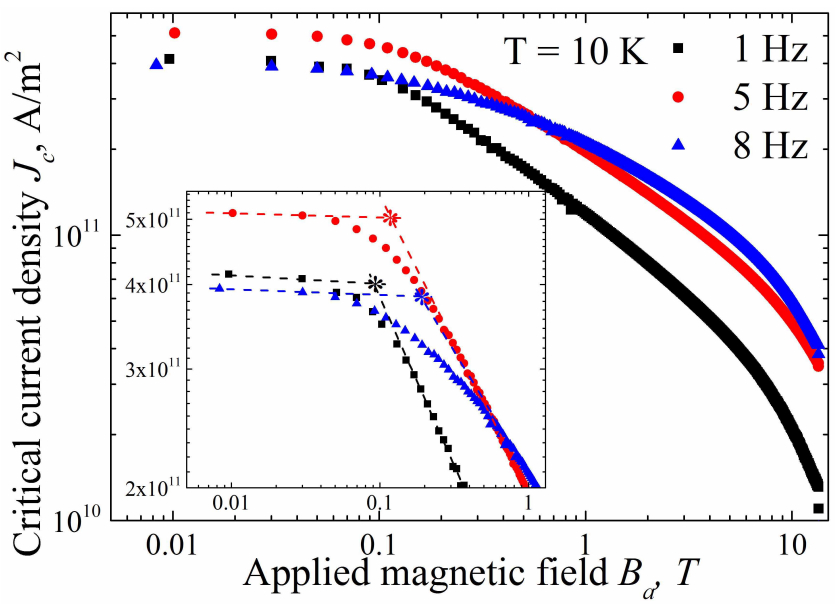

FIG. 7. Critical current density dependence on the applied magnetic field for the films deposited at different frequencies measured at $T=10 \mathrm{~K}$. Insert shows a magnification of the low field region. The characteristic fields $B^{*}$, determined at the crossing point of the line extensions from the two linear parts of the $J_{c}\left(B_{a}\right)$ dependences, are: $B^{*}(1 \mathrm{~Hz})=0.09 \mathrm{~T}$, $B^{*}(5 \mathrm{~Hz})=0.12 \mathrm{~T}, B^{*}(8 \mathrm{~Hz})=0.18 \mathrm{~T}$.

ber of pinning centres introduced during deposition at higher laser frequencies. In the inset to Fig. 7 , the low field region of $J_{c}\left(B_{a}\right)$ dependences at $10 \mathrm{~K}$ is shown, where the so-called characteristic field $B^{*}$ can be determined at the crossing of the extensions to the two linear regions according to Ref. ${ }^{61}$. This field, denoting transition from the single vortex pinning regime at low fields to a collective pinning regime at higher fields, can be used to accurately determine the pinning radius of the individual vortex pinning ${ }^{77,78}$. Note, $B^{*}$ can be defined as the field at which the critical current reaches the $90 \%^{79}$ or $95 \%^{77,78}$ of the value obtained for zero applied field. However, in this work it is sufficient to qualitatively demonstrate that $B^{*}$ is the transition from a plateau-like $J_{c}\left(B_{a}\right)$ dependence to the power-law dependence and is directly proportional to dislocation density (as well as to the island density at any thickness of the film $)^{61,77}$. The characteristic field $B^{*}$ increases with increasing laser frequency [Fig. 7] (note, this trend is independent of the $B^{*}$ definition). The $B^{*}$ increase indicates enhanced pinning and reduction of the individual depinning radius ${ }^{77,78}$. As $B^{*}$ is proportional to the dislocation density (dominating pinning in PLD YBCO films), it is natural to expect an enhancement of $J_{c}\left(B_{a}\right)$ consistent with the $B^{*}$ enhancement that is clearly the case, as can be seen in Fig. 7. It is also consistent with the increase of the island density [best seen in Fig. 5(a,c)], which is directly proportional to the corresponding increase of the dislocation density ${ }^{76}$ because dislocation formation predominantly occurs close to the boundary of islands ${ }^{64,80}$. The quantitative agreement between densities of dislocations and islands, as well as $B^{*}, J_{c}\left(B_{a}\right)$ and $J_{c}(0)$ may be not easy to find ${ }^{77}$ because while $B^{*}$ is determined by density of pinning centres in single vortex pinning regime, $J_{c}(0)$ is established by strength of individual pinning $\operatorname{cites}^{28}$. In addition, other effective pinning defects ${ }^{77,78}$, such as twin boundaries ${ }^{26,81}$, oxygen vacancies ${ }^{82}$ and $\mathrm{Y}_{2} \mathrm{O}_{3}$ inclusions ${ }^{83,84}$ are present and can be affected by deposition frequency. Indeed, smoother transitions at the higher deposition frequencies from the plateau-like $J_{c}\left(B_{a}\right)$ dependence at $B_{a} \rightarrow 0$ to the powerlaw dependence at the larger fields [insert in Fig. 7] indicate a broader distribution of available pinning sites.

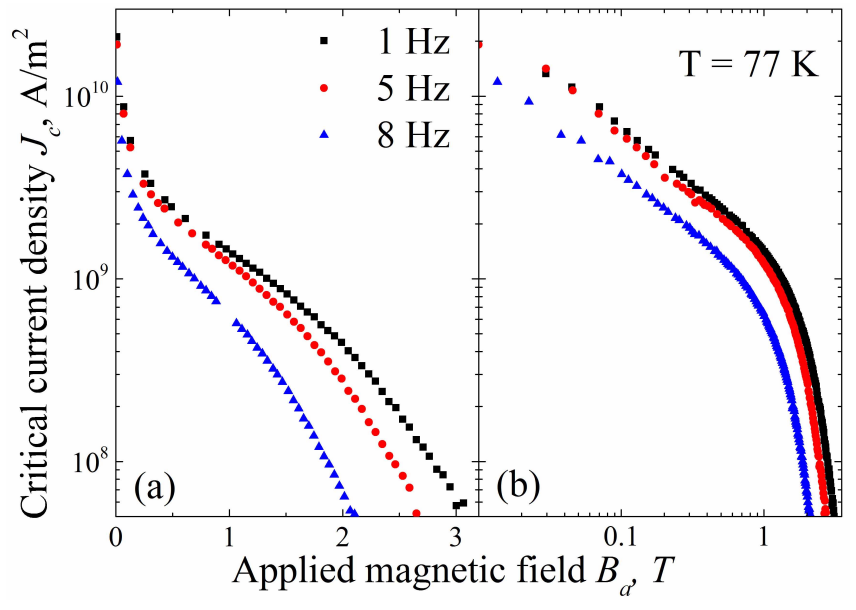

FIG. 8. Critical current density dependence on the applied magnetic field for the films deposited at different frequencies measured at $T=77 \mathrm{~K}$ in $\log$ (a) and $\log -\log$ (b) scale.

It is striking to compare $J_{c}$ curves measured at $10 \mathrm{~K}$ and $77 \mathrm{~K}$ [(Figs. 7 and 8]. The trend of the deposition frequency dependent $J_{c}\left(B_{a}\right)$ curves at $77 \mathrm{~K}$ is reversed. Variation of $J_{c}\left(B_{a}\right)$ at $77 \mathrm{~K}$ with deposition frequency can be conveniently demonstrated with irreversibility field which increases from $\sim 2 \mathrm{~T}$ to $\sim 3 \mathrm{~T}$ by decreasing $f$ from $8 \mathrm{~Hz}$ and $1 \mathrm{~Hz}$ (Fig. 8(a)). Remembering the structural feature changes associated with the changing the deposition frequency, which also results in the observed $T_{c}(f)$ behaviour (Fig. 6), the scenario of this reversal behaviour can be as follows. The coherence length increases as a function of temperature. This means that the effectiveness of various pinning sites changes with temperature. Furthermore, it is obvious that the longer the coherence length, the more transparent the structure of the films is for the current flow. This is valid only as long as the structure-induced reduction of the superconducting order parameter at the defects is shorter than the coherence length. Thus, having our main assumption that dislocations provide the most effective pinning in YBCO PLD films, it is straightforward to correlate higher $J_{c}\left(B_{a}\right)$ curves at low temperatures in Fig. 7 with a larger number of dislocations, while the transparency of the films to the supercurrent flow is not substantially affected. This is also qualitatively in agreement with the results obtained in Ref. ${ }^{35}$. There, stronger $J_{c}$ enhancements observed at low temperatures is related 
to a higher dislocation density induced in the films by the artificial multilayered structure and the corresponding additional misfit-driven relaxation of the crystal structure through formation of additional dislocations. It is possible that at higher temperatures, the pinning dominance of the dislocations smears out by the presence of other structural features, as well as thermal activations. Collectively, these additional factors weaken the dislocation-based pinning model, and reverse the $J_{c}\left(B_{a}\right)$ trend so that the films with higher densities of dislocations obtained at higher frequencies show lower $J_{c}\left(B_{a}\right)$ curves at high temperatures compared to the films with lower dislocation densities deposited at lower frequencies.

These temperature/field/frequency dependencies provide enormous possibilities for tuning YBCO thin film properties for particular applications. Indeed, at high magnetic fields, the films deposited at higher frequencies perform better at low temperatures and worse at higher temperatures. At the same time, at the low field region (self-field region), the $5 \mathrm{~Hz}$ film exhibits the most optimal properties at low temperatures. These differences are likely the ground for existing inconsistencies in determining the pinning mechanism in the PLD YBCO films, ranging from dislocation dominance $28,29,61,85$ and twin boundaries $^{81}$ to small non-superconducting inclusions of $\mathrm{Y}_{2} \mathrm{O}_{3}{ }^{83,84}$ and even surface pinning ${ }^{86}$ and Josephson-like weak-links ${ }^{79}$, which may simply represent different but limited aspects of the entire picture, as shown in this work.

\section{SUMMARY}

In summary, hard ferromagnetic FePt and high temperature superconducting YBCO thin films have been fabricated by PLD. It has been shown that the structure of these films, belonging to completely different classes of films with different growth mechanisms and different physical properties, can effectively and easily be controlled by the laser frequency upon the deposition process. This approach offers not only a simple technological solution to existing problems of loosing useful detectable signal associated with reducing film thicknesses, but also the possibility to design required physical properties at will via the accurate control of the corresponding film structure.

In the case of the FePt films, the laser frequency affects the ratio of the out-of-plane to the in-plane growth rates, allowing practically independent island growth in the in-plane and the out-of-plane directions. As a result of laser frequency change from $1 \mathrm{~Hz}$ to $2 \mathrm{~Hz}$, the film structure evolves from the percolated to elongated islands. A further laser frequency increase from $2 \mathrm{~Hz}$ to $6 \mathrm{~Hz}$ decreased the island size by a factor of 2 . Thus, we are able to significantly increase the coercive field (by a factor of 20 , from $0.2 \mathrm{~T}$ to $4.6 \mathrm{~T}$ at room temperature) by very moderate and practical changes of the laser frequency. At the same time, the dipole-dipole interaction between ferromagnetic islands, being proportional to island size, is halved if the frequency is increased from $2 \mathrm{~Hz}$ to $6 \mathrm{~Hz}$. MFM study has confirmed the structure-driven coercive field dependence on the laser frequency. The films deposited with $2 \mathrm{~Hz}$ and $6 \mathrm{~Hz}$ consist of single magnetic domain islands, and their demagnetization process is governed by spontaneous flip of the magnetic moment, which, together with high magnetic anisotropy, leads to high coercive fields in these films. On the contrary, due to the percolated structure of the $1 \mathrm{~Hz}$ film the demagnetization process is dominated by magnetic domain wall formation and propagation, which leads to low coercive fields despite high magnetic anisotropy. The results become even more spectacular if we indicate the fact that the films have been deposited in a relatively low vacuum. On one hand, the low vacuum may reduce the technological cost of film production if necessary films parameters can be met; on the other hand, a higher vacuum can significantly broaden the range of accessible films characteristics to achieve growing industry demands.

The YBCO films possess more sophisticated response to structural changes of the films as a result of laser frequency variation, compared to FePt thin films. It is obviously due to different growth mode and more sophisticated chemical composition of the YBCO films. AFM studies revealed that the island density increased if the frequency was varied from $1 \mathrm{~Hz}$ to $8 \mathrm{~Hz}$. Laser frequency increase promotes the in-plane island growth and surface roughness/area decrease. The effective manipulation of the superconducting properties has been achieved by varying the deposition frequency from $1 \mathrm{~Hz}$ to $8 \mathrm{~Hz}$. The $T_{c}$ transition and its width can be maximised by reducing the deposition frequency down to $1 \mathrm{~Hz}$. The density of the pinning sites, described by the characteristic field $B^{*}$, is maximized by increasing the deposition frequency. The increased pinning centres density (obtained at $8 \mathrm{~Hz}$ ) also enhance the critical current density at low temperatures and high magnetic fields. At the same time, the higher superconducting critical temperature (achieved at lower deposition frequencies) lead to higher $J_{c}\left(B_{a}\right)$ at high temperatures. In the self-field region, the most optimal conditions for the $J_{c}$ performance are obtained at $5 \mathrm{~Hz}$.

Despite the difference of film growth modes between FePt and YBCO in both cases the laser frequency controls the structure of films by affecting in-plane to out-ofplane growth rates of islands. The out-of-plane growth of FePt islands (genuine islands) is promoted by higher deposition frequencies, increasing the island density. In contrast, the out-of-plane growth of YBCO islands (i.e., $3 \mathrm{D}$ stack of terraces) is promoted by low frequencies. The high deposition frequencies suppress 3D islanding and stimulate layer-by-layer growth of YBCO, but increase the density of islands (which are two dimensional or at least demonstrate a lesser degree of $3 \mathrm{D}$ islanding) in the case of FePt.

All of these physical property manipulations were achieved in the films with a similar thickness as a result 
of the structural modification response to the deposition frequency variation. The frequency of PLD technique emerges as a powerful and promising tool for effective tuning the physical properties in thin films of different types, having in mind their particular applications.

\section{ACKNOWLEDGMENT}

This work is supported by the Australian Research Council via A. V. Pan's Discovery Projects (DP0879933 and DP110100398), as well as by HYPRES, Inc. (USA). Authors acknowledge the Australian National Fabrication Facility (ANFF) and UOW Electron Microscopy Centre for providing instrumentation.
[1] T. Shima, K. Takanashi, Y. K. Takahashi, K. Hono, Appl. Phys. Lett. 85, 2571 (2004)

[2] T. Shima, K. Takanashi, Y. K. Takahashi, K. Hono, Appl. Phys. Lett. 81, 1050 (2002)

[3] A. A. Baski, H. Fuchs, Surface Science 313, 275-288 (1994)

[4] S. V. Pysarenko, A. V. Pan, S. X. Dou, IEEE Trans Appl. Supercond. 21, 3179 (2011).

[5] Foltyn S R et al 2007 Nature Mater. 6631.

[6] S. Pysarenko, A. V. Pan, and S. X Dou, Influence of thickness and deposition rate on superconducting properties of $\mathrm{YBa}_{2} \mathrm{Cu}_{3} \mathrm{O}_{7}$ films, Proc. 30th Annual A\&NZIP Condensed Matter and Materials Meeting, Ed. M. Avdeev, Aust. Inst. Phys. (AIP 2006). ISBN 1920791-09-4

[7] P. Jensen, B. Niemeyer, Surf. Sci. 384, L823-L827 (1997)

[8] N. Combe, P. Jensen, Phys. Rev. B 57, 15553 (1998)

[9] J. M. Warrender, M. J. Aziz, Phys. Rev. B 76, 045414 (2007)

[10] M. J. Aziz, Appl. Phys. A 93, 579 (2008)

[11] I. A Golovchanskiy, S. A Fedoseev and A. V. Pan, J. Phys. D: Appl. Phys. 46 (2013) 215502

[12] J.A. Vanables, G.D.T. Spiller, M. Hanbucker, Rep. Prog. Phys. 47, 399 (1984)

[13] S.J.Pennycook et.al., Physica C: Supercond 202, 1 (1992)

[14] C.M. Gilmore, J.Kim, Appl. Phys. A 75, 565 (2002)

[15] H.J. Scheel, M. Berkowski and B. Chabot, Journal of Crystal Growth 115(1991) 19.

[16] P. Chaudhari, R. H. Koch, R. B. Laibowitz, T. R. McGuire, and R. J. Gambino, Phys. Rev. Lett. 58, 2684 (1987).

[17] G. W. Crabtree, J. Z. Liu, A. Umezawa, W. K. Kwok, C. H. Sowers, S. K. Malik, B. W. Veal, D. J. Lam, M. B. Brodsky, and J. W. Downey, Phys. Rev. B 36, R4021 (1987).

[18] D. Larbalestier, A. Gurevich, D. M. Feldmann and A. Polyanskii, Nature 414, 3682001

[19] F.C. Wellstood, J.J. Kingstone, J. Clarke, J. Appl. Phys. 75,683 (1994)

[20] X. Chaud et al., IEEE Trans. Appl. Supercond. 22(3), 2012

[21] G.-C. Liang et al., IEEE Trans. Microw. Theory Tech. 43, 3020 (1995)

[22] H. Chapoupka et al., Electron. Lett. 32, 1735 (1995)

[23] K.E. Andreev et al., Supercond. Sci. Tech. 10, 366 (1997)

[24] Y.H. Li et al., Physica C 265, 328 (1996)

[25] L. Civale et al., Phys. Rev. Lett. 67 (1991) 648.
[26] G. Blatter et al., Rev. Mod. Phys. 66 (1994) 1125.

[27] V. M. Pan and A. V. Pan, Fiz. Niz. Temp. 27 (Rus.), 991 (2001) [Low Temp. Phys. 27, 732 (2001)].

[28] V. Pan et.al., Phys. Rev. B 73, 054508 (2006).

[29] I. A. Golovchanskiy, A. V. Pan, O. V. Shcherbakova, S. A. Fedoseev and S. X. Dou, Supercond. Sci. Technol. 24, 105020 (2011).

[30] I. A. Golovchanskiy, A. V. Pan, S. A. Fedoseev, O. V. Shcherbakova, S. X. Dou, Physica C 479, 151 (2012).

[31] E. Osquiguil, M. Maenhoudt, B. Wuyts, and Y. Bruynseraede, Appl. Phys. Lett. 60, 1627 (1992)

[32] Jinhua Ye and Keikichi Nakamura, Phys. Rev. B 48, 7554 (1993)

[33] A. V. Pan, S. V. Pysarenko, D. Wexler, S. Rubanov, and S. X. Dou, IEEE Trans. Appl. Supercond. 17, 3585 (2007).

[34] C. C. Chang et al., Appl. Phys. Lett. 57, 1814 (1990).

[35] A. V. Pan, S. Pysarenko, and S. X. Dou, Appl. Phys. Lett. 88, 232506 (2006).

[36] M.H. Kryder, E.C. Gage, T.W. McDaniel, W.A. Challener, R.E. Rottmayer, G. Ju, Y.-T. Hsia, M. Fatih Erden, Proceedings of the IEEE 96, 1811 (2008); Seagate demonstration in The Magnetic Recording Conference, USA, 2012

[37] S. Fahler, V. Neu, M. Weisheit, U. Hannemann, S. Leinert, A. Singh, A. Kwon, S. Melcher, B. Holzapfel, L. Schultz in (18th Workshop on High Performance Magnets and their Applications, Annecy, France, 2004)

[38] S. D. Bader, Reviews of Modern Physics 78, 1 (2006)

[39] S. Haindl, M. Weisheit, T. Thersleff, L. Schultz, B. Holzapfel, Supercond. Sci. Technol. 21, 045017 (2008)

[40] A. Moser, K. Takano, D. T. Margulies, M. Albrecht, Y. Sonobe, Y. Ikeda, Sh. Sun, E. E. Fullerton, J. Phys. D: Appl. Phys. 35, R157-R167 (2002)

[41] D. Weller, A. Moser, L. Folks, M. E. Best, W. Lee, M. F. Toney, M. Schwickert, J. Thiele, M. F. Doerner, IEEE Trans. Magn. 36, 10 (2000)

[42] H. Zhou, H. N. Bertram, IEEE Trans. Magn. 35, 2712 (1999)

[43] Yu. A. Genenko, et al., Appl. Phys. Lett. 84, 3921 (2004).

[44] Yu. A. Genenko, A. V. Snezhko, and H. C. Freyhardt, Phys. Rev. B 62, 3453 (2000).

[45] A. V. Pan, S. Zhou, H. Liu, S. Dou, Supercond. Sci. Technol. 16, L33 (2003)

[46] A. V. Pan, S. Dou, J. Appl. Phys. 96, 1146 (2004)

[47] R. F. C. Farrow, D. Weller, R. F. Marks, M. F. Toney, S. Hom, G. R. Harp, A. Cebollada, Appl. Phys. Lett. 69, 
$1166(1996)$

[48] Y. Endo, N. Kikuchi, O. Kitakami, Y. Shimada, J. Appl Phys. 89, 7065 (2001).

[49] T. Suzuki,K. Harada, N. Honda, K. Ouchi, Journal of Magnetism and Magnetic Materials 193, 85 (1999).

[50] Y. Wang et al., Appl. Surf. Sci. 288, 381-391 (2014).

[51] S. Fahler, U. Hannemann, M. Weisheit, V. Neu, S. Melcher, S. Leinert, S. C. Wimbush, A. Singh, A. Kwon, B. Holzapfel, L. Schultz, Appl. Phys. A 79, 1529-1531 (2004).

[52] M. Weisheit, L. Schultz, S. Fahler, J. Appl Phys. 95, 7489 (2004).

[53] X. H. Li, B. T. Liu, W. Li, H. Y. Sun, D. Q. Wu, X. Y. Zhang, J. Appl. Phys. 101, 093911 (2007)

[54] M. H. Hong, K. Hono, M. Watanabe, J. Appl. Phys. 84, 4403 (1998)

[55] M. Weisheit, L. Schultz, S. Fahler, Journal of Magnetism and Magnetic Materials 290-291, 570-572 (2005)

[56] I. A. Golovchanskiy, A. V. Pan, O. V. Shcherbakova, and S. A. Fedoseev, J. Appl. Phys. 114, 163910 (2013)

[57] A. V. Pan, I. A. Golovchanskiy, and S. A. Fedoseev, Europhys. Lett. 103, 17006 (2013).

[58] D.-X. Chen and R. B. Goldfarb, J. Appl. Phys. 66, 2489 (1989)

[59] Guoqing Li, Hideaki Takahoshi, Hirotaka Ito, Takahiro Washiya, Hitoshi Saito, Shunji Ishio, Toshiyuki Shima, Koki Takanashi, Journal of Magnetism and Magnetic Materials 287 (2005) 219.

[60] T. Shimaa, K. Takanashi, Y.K. Takahashi, K. Hono, G.Q. Li, S. Ishio, Journal of Magnetism and Magnetic Materials 266 (2003) 171.

[61] B. Dam et al., Nature 399, 439 (1999)

[62] M. Hawley et al., Science 350, 1587 (1991)

[63] C. Gerber et al., Nature 350, 279 (1991)

[64] X.-Y. Zheng et al., Phys. Rev. B 45, 7584 (1992)
[65] B. Dam, J. M. Huijbregtse, J. H. Rector, Phys. Rev. B 65, 064528 (2002)

[66] S. R. Foltyn, Q. X. Jia, P. N. Arendt, L. Kinder, Y. Fan, and J. F. Smith, Appl. Phys. Lett. 75, 3692 (1999).

[67] D. J. Srolovitz, Arta metal 37, 621 (1989).

[68] G.A. Farnan et al., Supercond. Sci. Tech 13, 262 (2000)

[69] F. Wellhofer et al., Appl. Surf. Sci 127129, 525530 (1998).

[70] P. Muller, R. Kern, Appl. Surf. Sci. 102, 6-11 (1996)

[71] B. Dam, J.H. Rector, J.M. Huijbregtse, R. Griessen, Physica C 305, 1 (1998)

[72] D.B. Geohegan, Appl. Phys. Lett. 60, 2732 (1992).

[73] M.R. Predtechensky, A.P. Mayorov, Appl. Supercond. 1, 2011 (1993).

[74] A. Eulenburg et al., Physica C 312, 91 (1999).

[75] V. M. Pan et al., Cryogenics 33, 21 (1993)

[76] J. M. Huijbregtse et al., Phys. Rev. B 62, 1338 (2000)

[77] A. V. Pan, et al., Physica C 407, 10 (2004).

[78] A. V. Pan and S. X. Dou, Phys. Rev. B 73, 052506 (2006).

[79] E. Mezzetti, R. Gerbaldo, G. Ghigo, L. Gozzelino, B. Minetti, C. Carmelingo, A. Monaco, G. Cuttone, and A. Rovelli, Phys. Rev. B 60, 7623 (1999).

[80] V. Svetchnikov, V. Pan, C. Traeholt, H. Zandbergen, IEEE Trans. Appl. Supercond. 7, 1396 (1997).

[81] P. Paturi et al., J. Appl. Phys. 105, 023904 (2009).

[82] M.Daeumling et al., Nature 346, 332 (1990)

[83] C. J. van der Beek, et al., Phys. Rev. B 66, 024523 (2002).

[84] A. E. Koshelev, A. B. Kolton, Phys. Rev. B 84, 104528 (2011).

[85] F. C. Klaassen, G. Doornbos, J. M. Huijbregtse, R. C. F. van der Geest, B. Dam, and R. Griessen, Phys. Rev. B 64, 184523 (2001).

[86] V. A. Khokhlov, A. I. Kosse, Yu. E. Kuzovlev, G. G. Levchenko, Yu. V. Medvedev, A. Yu. Prokhorov, P. Mikheenko, R. Chakalov, and C. M. Muirhead, Supercond. Sci. Technol. 17, S520 (2004). 